Derleme / Review

\title{
Çocuk Hastalarda Telerehabilitasyon ve Pediatri Hemşiresinin Sorumlulukları
}

\section{Telerehabilitation in Children Patients and the Responsibilities of Paediatric Nurse}

\author{
Atiye Karakul ${ }^{a^{*}}$ \\ a Dr.Öğretim Üyesi, Tarsus Üniversitesi, Sağlık Bilimleri Fakültesi Hemşirelik Bölümü, Hemşirelik Anabilim Dalı, Mersin, Türkiye, ORCID: https://orcid.org/0000-0001-6580-9976 \\ "Iletişimden sorumlu yazar, E-mail: atiyekarakul@gmail.com
}

\section{ARTICLE INFO}

Article History

Received 27.06.2021

Received in revised form 14.07.2021

Accepted 19.07.202

Keywords:

Tele-rehabilitation

Tele-nursing

Pediatric

\begin{abstract}
Tele-nursing is defined as "the use of communication technologies in nursing to improve patient care". Among the tele-nursing practices; there are some other terms such as tele-pediatric, tele-rehabilitation, tele-triage, tele-trauma, tele-psychology, tele-cardiology, tele-home care, tele-forensic care and tele-intensive care. Pediatric nurses make use of the electronic information and communication technology of the tele-rehabilitation service, enabling patients and caregivers to access rehabilitation services remotely. In particular, it facilitates the continuity of education. Continuity of follow-up is ensured through regular communication. Through this system, it is easier to monitor the progress in tele-rehabilitation continuously and to make changes in the treatment program immediately. Therefore, pediatric nurses should support their roles and responsibilities in the telerehabilitation process. In addition, it is recommended to organize seminars and workshops to increase awareness among pediatric nurses.
\end{abstract}

(C) 2021 Mardin Artuklu University. All rights reserved.

\section{MAKALE BİLGILERI}

Makale Geçmişi:

Geliş Tarihi: 27.06.2021

Revizyon Tarihi: 14.07 .202

Kabul Tarihi: 19.07.2021

Anahtar Kelimeler:

Telerehabilitasyon

Telehemşirelik

Pediatri

\section{ÖZET}

Tele-hemşirelik; "Hasta bakımını geliştirmek için hemşirelikte iletişim teknolojilerinin kullanılmasıdır" şeklinde tanımlamaktadır. Tele-hemşirelik uygulamaları arasında; tele-pediatrik, tele-rehabilitasyon bașta olmak üzere teletriyaj, tele-travma, tele-psikoloji, tele-kardiyoloji, tele-evde bakım, tele-adli bakım ve tele-yoğun bakım yer almaktadır. Pediatri hemşireleri tele-rehabilitasyon hizmetinin elektronik bilgi ve iletişim teknolojisinden yararlanarak hastaların, bakım vericilerin rehabilitasyon hizmetine uzaktan erişmesini sağlamaktadır. Özellikle eğitimin devamlılığının sağlanmasını kolaylaştırmaktadır. Düzenli olarak iletişimde bulunulması yoluyla izlemin de sürekliliği sağlanmaktadır. Bu sistem aracılığıyla telerehabilitasyondaki gelișimin sürekli olarak izlenebilmesi ve tedavi programında değişikliklerin hemen yapılabilmesini kolaylaşmaktadır. Bu nedenle pediatri hemşireleri telerehabilitasyon sürecindeki rol ve sorumluluklarını desteklemelidir. Ek olarak pediatri hemşireleri arasında farkındalığı artırmak için seminer ve çalıştayların düzenlenmesi önerilmektedir.

(C) 2021 Mardin Artuklu Üniversitesi. Tüm hakları saklıdır.

\section{Giriş}

Tele-sağlık günümüzde, telefon ve internet dahil telekomünikasyon teknolojisini kullanarak uzaktan izlem ile tedavi planları, hasta ve bakım verici eğitimi, danışmanlık ve takip etme süreci olarak tanımlanır (1). Dünyadaki tarihsel süreci 1940'lı yıllarda tele-konsültasyon ve tele-evde bakım ile başlamıştır.1960'larda NASA astronotları için yaşamsal belirtileri ileten tele-tıp veya tele sağlık uygulamaları gerçekleşmiştir. 1970'lerde gerçek tıbbi uygulamalara dönüşmüştür. 1980’lerde ise ordu da kullanılmaya başlamıştır. Uzmana erişim için konferans kullanılmıştır. 1990'larda ise federal kurumlar tele-sağlık programlarını kullanmaya başlamıştır. Türkiye'de ise bu süreç 2000'li yıllarda ilk gündeme gelmeye başlamıştır. 2006'da eylem planı hazırlanmış olup 2007 yilında tele-radyoloji, tele-pataloji ve tele-EKG servisleri kurulmuştur. 2008'de uygulama kapsamında hastane sayısında artış olmuştur. Ayrıca hastane ve birinci basamak sağlık hizmetleri arasında web site kurulmuştur. Sağlık Bakanlığı evde sağlık bakım hizmetini ise 2010 yılından itibaren vermeye başlamıştır $(2,3)$. 
Tele-sağlık evde, sağlık kliniklerinde, hastanelerde ve hapishanelerde uygulanmaktadır. Özellikle telefonla triyaj, uzaktan izleme ve evde bakım hızla büyüyen uygulamalardandır. Tele-sağlıktaki büyüme, Tele-hemşirelik uygulama alanın oluşmasını sağlamıştır (4).

Tele-hemşirelik; tele-sağllğın bir alt kümesi olarak belirtilmektedir. Teknoloji, hemşirelik bakımını sağlamak ve hemşirelik uygulamaları yürütmek için kullanılmaktadır. Uluslararası Hemşireler Birliği (ICN) "Hasta bakımını geliştirmek için hemşirelikte iletişim teknolojilerinin kullanılmasıdır" șeklinde tanımlamaktadır. Tele-hemşirelik uygulamaları arasında; tele-pediatrik, tele-rehabilitasyon, teletriyaj, tele-travma, tele-psikoloji, tele-kardiyoloji, tele-evde bakım, tele-adli bakım ve tele-yoğun bakım yer almaktadır $(4,5)$. Telerehabilitasyon, "Rehabilitasyon hizmetinin elektronik bilgi ve iletişim teknolojisinden yararlanarak uzak mesafelere iletilmesi” olarak tanımlanmaktadır (1). Telerehabilitasyon sistemi, rehabilitasyon hizmetinin elektronik bilgi ve iletişim teknolojisinden yararlanarak hastaların, bakım vericilerin rehabilitasyon hizmetine uzaktan erişmesini sağlamaktadır. Dolayısıyla verilen eğitimin devamlılığı sağlanması kolaylaşmaktadır. Düzenli olarak iletişiminde bulunularak izlemin de sürekliliği olmaktadır. Hasta eğitiminde ve rehabilitasyonunda devamlılık, rehabilitasyondaki gelişimin izlenebilmesi, tedavi programında değişikliklerin yapılabilmesi, çocuk ev ebeveynlerin yolda geçirdiği zaman ve harcamadan tasarruf gibi faydalar edinilebilmektedir. Telerehabilitasyon hizmetinin iletişim teknolojilerinden; Telefon ile olabileceği gibi alınan verileri temel alarak, mobil akıllı telefon tabanlı bir uygulamaya dahil olan e-posta, SMS ve mesajlar aracılığıyla da hastalara özel geri bildirim sağlama olanağı bulunmaktadır (7-11).

Telerehabilitasyonun iki evresi vardır. Bunlardan birincisi başlangıç evresi olup, ikincisi ise temel (ev) evredir. Başlangıç evresi, Klinik değerlendirme, eğitim, bireysel egzersiz eğitiminin planlanması ve gözlem altında taburcu oluncaya kadar eğitim seanslarını içermektedir. Temel evre; Eğitim öncesi mobil telefonla verilerin iletilmesi, kontrendikasyon yoksa eğitimin başlaması, görüşme sonrası hastanın sorularının dinlenmesi, özel rehabilitasyon programının etkinliğinin ve doğruluğunun değerlendirilmesini içermektedir (3).

\subsection{Telerehabilitasyon Uygulamalarının Avantaj ve Dezavantajları}

Telerehabilitasyon uygulamalarının hem avantajları hem de dezavantajları bulunmaktadır. Şekil 1'de telerehabilitasyon uygulamalarının avantajları gösterilmektedir.

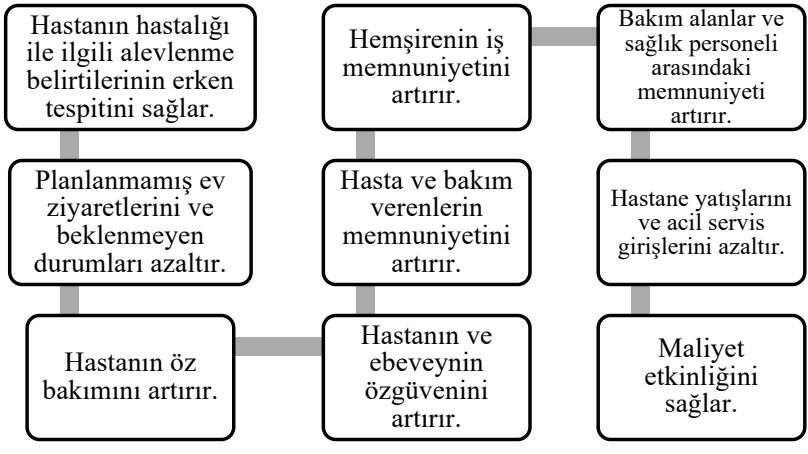

Şekil 1 - Telerehabilitasyon uygulamalarının avantajları (4,1218).

Telerehabilitasyon uygulamalarının dezavantajlarına ise Şekil 2 'de yer verilmektedir.

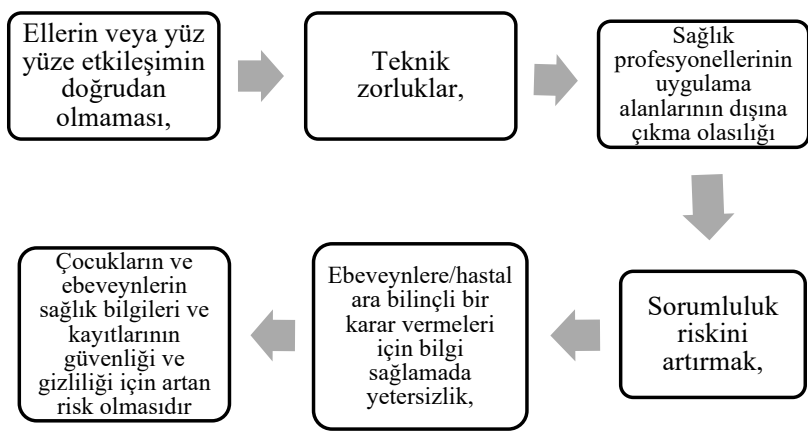

Şekil 2 - Telerehabilitasyon uygulamalarının dezavantajları $(4,15)$

\subsection{Telerehabilistayon Uygulamalarında Pediatri Hemşiresinin Rolü}

Pediatrik popülasyonlar genellikle kısa ilgi süreleri ve teknik sorunlara karşı düşük tolerans sergilemektedir (özellikle de sanal rehabilitasyon müdahalesinin ilk yeniliği ortadan kalktığında). Ev ortamı, hastayı motive etmede ve desteklemede önemli bir rol oynar ve tele-rehabilitasyon uygulamasının etkinliğinin sağlanmasında katkısı oldukça büyüktür. Telerehabilitasyon görüşmelerinin süresinin ve sıklığının sürdürülebilir olmasını sağlamak (teknolojik sınırlamalar da dikkate alındığında) için bakım vericinin desteği yadsınamayacak kadar önemlidir. Bakım vericiden alınan destek sayesinde hasta katılımının aktif olmasını ve bakımın sürdürülebilir olması kolaylaşmaktadır. Telerehabilitasyon uygulamalarında pediatri hemşiresinin sorumluluklarından bir tanesi eğitimdir. Dolayısıyla pediatri hemşiresi, eğitimi planlarken hastanın ve ailenin gereksinimlerini dikkate alması gerekmektedir. Gereksinimleri belirledikten sonra gerekli eğitimleri planlamalı, hazırlıkları yapması önerilmektedir. 
Pediatri hemşiresi eğitimi planlar ve hazırlarken özellikle elektronik ortamda hem ebeveynin eğitim seviyesi hem de çocukların gelişim düzeyine uygun ve doğru dokümantasyonu sağlaması önem taşımaktadır. Hasta ve yakınlarına ait bilgilerin gizliliğini ve güvenliğini sağlama, bakım vermek için hastaebeveyn-hemşire ilişkisi kurmak, hasta ve ebeveynin manevi ve psikososyal ihtiyaçlarını/tercihlerini dikkate almak gerekmektedir. Ek olarak hasta ve ebeveynin bilgilendirilmiş onayını sağlamak pediatri hemşiresinin sorumlulukları arasında yer almaktadır. Pediatri hemşiresi, bu süreci planlar ve uygularken mevcut kurumsal politikalara ve yönergelere uymaya dikkat etmesi önem arz etmektedir. Bu nedenle sadece yapmaya yetkili olunan hemşirelik girişimlerini gerçekleştirmek oldukça önemlidir $(4,5,19,20)$. Bu süreçte meydana gelebilecek yasal/etik sorunlara pediatri hemşiresinin hassasiyet göstermesi gerekmektedir. Telerehabilitasyon uygulamalarında pediatri hemşiresi hesap verme, güvenlik, mahremiyet ve gizlilik (sağlı bilgilerini korumak için şifrelemeli uygulamalar, özel ofis kullanmak, odadaki tüm kişilerin tanınması), bilgilendirilmiş onay vermek ve sorumluluk sık karşılaşılan yasal/etik konulardır.

$\mathrm{Bu}$ nedenle pediatri hemşirelerine, bu uygulamalar için hasta ve ailesi için verilen elektronik hesapların ya da uygulamaların güvenliğinin sağlanması için gerekli tedbirleri alması/tedbirlerin sağlanması önerilmektedir. Mümkünse mahremiyeti sağlamak için görüşmelere varsa ayrı ofiste yoksa ayrı odalarda yapması pediatri hemşiresine kolaylık sağlayabilmektedir (20). Gagnon et al. (10) 8-21 yaş arası artrogripozis tanılı 10 çocuk ile 12 haftalık bireyselleştirilmiş ev temelli rehabilitasyon uygulaması yaptıkları çalışmada, çocukların fiziksel aktivite ve fonksiyon düzeylerinin arttığı, ağrılarının ise azaldığını belirtmiştir. Lawend et al. (22) çalışmasında, astım tanılı 10-18 yaş 60 çocuğa telerehabilistasyon uygulaması olarak anneleri tarafindan masaj terapi uygulatılmıştır. Çalışma sonucunda, çocukların anksiyete seviyesinde azalma görülürken memnuniyet düzeyinde ise artma görülmüştür. Steinhart et al. (17), myelomeningosel tanılı 14-18 yaş arası 4 adölesan ile video konferans ile günlük takip uyguladığı çalışmada, adölesanların bilişsel oryantasyon düzeyinin, fonksiyonel durumunun ve yaşam kalitesinin attı̆̆ saptanmıştır. Ek olarak hastalar uygulamadan memnun kaldıklarını ve özgüvenlerinin arttığını belirtmiştir. Ülkemizde de telerehabilitasyon uygulamasına yönelik birçok çalışma bulunmaktadır. Çalışmalar, hastaların yaşam kalitesini artırmada ve stresi azaltmada, telerehabilitasyon uygulamalarının etkin olduğunu ortaya koymuştur (23). Tutar Güven (24) çalışmasında epilepsi tanılı adölesanlara Web Tabanlı Epilepsi Eğitim Programı uygulamıştır. Çalışma sonucunda, uygulamanın adölesanların bilgi düzeyinde artışı sağladığına ve öz yeterliliğini arttığına ulaşılmıştır. Çalışmalar telerehabilitasyon uygulamasını yaparken pediatri hemşiresinin, hasta ve ailesine verilmesi gereken standart bakım değişmeyip sadece sonuca ulaşma yöntemi farklı olduğunu ortaya koymuştur. Pediatri hemşiresi de bu durumu çocuk hasta ve ailesine belirtmesi gerekmektedir.

\section{Sonuç ve Öneriler}

Sonuç olarak, insan faktörleri ve teknoloji kullanılabilirliği, genel olarak tele-sağlık programlarının kullanımında, kabulünde ve ekonomik uygulanabilirliğinde önemli rol oynamaktadır. Bu nedenle, yeni programların planlanmasına ve uygulanmasına ve yeni cihazların tasarımına insan faktörleri ve kullanıcı merkezli tasarım teorilerinin uygulanması esastır. Bu insan faktörlerini dikkate alan son tele-sağlık uygulama standartları ve teknik kılavuzlarla desteklenmektedir. İnsan faktörleri ilkeleri, telerehabilitasyonun büyümesinin de merkezindedir. Telerehabilitasyon hizmet sunumunun tüm yönlerinin, yaşlarına, geçmişlerine veya yeteneklerine bakılmaksızın tüm kişiler tarafından erişebilir, verimli, anlaşılabilir ve kullanılabilir olması oldukça önemlidir. Pediatri hemşireleri telerehabilitasyon sürecindeki rol ve sorumluluklarını desteklemelidir. Pediatri hemşireleri ve sağlık çalışanları arasında farkındalığı artırmak için seminer ve çalıştaylar düzenlenmelidir. Düzenlenen bu eğitimlerin belirli aralıklarla tekrarlanması ve değerlendirilmesi önerilmektedir. Bakım sürecinin yönetiminde rehberlerin ve protokollerin hem hasta hem de ailenin gereksinimlerinin dikkate alınması önemlidir. Son olarak hükümet ülkedeki telerehabilitasyon uygulamalarını desteklemeli ve gerekli yasal düzenlemeler yapılmalıdır.

Çıkar Çatışması: Herhangi bir kişi ve/veya kurum ile ilgili çıkar çatışması yoktur.

Finansal Destek: Bu makalede herhangi bir nakdî/ayni yardım alınmamıştır

Etik Kurul Onayı: Bu makale derleme türünde yazıldığı için etik kurul onayına gerek yoktur. 


\section{Kaynaklar}

1. Kalyon TA, Telerehabilitasyon, Fiziksel Tip 1999; 2 (2): 53-58

2. Brennan DM, Barker LM. Human factors in the development and implementation of telerehabilitation systems. Journal of Telemedicine and Telecare.2008; 14(2): 5558.

3. Akıncı B, Zenginler Y, Tele-rehabilitasyon. Turkiye Klinikleri J Physiother Rehabil-Special Topics, 2015;1(1):14-21

4. Pazar B, Taştan S, İyigün E. Tele sağlık sisteminde hemşirenin rolü. Bakırköy Tıp Dergisi.2015;11(1): 1-4.

5. https://www.icn.ch/ [Internet].Available from: http://www.icn.ch/networks/teleaims-and-objectives/ (Erişim tarihi 15.12.2020).

6. Ersoy S, Yıldırım Y, Şenuzun Aykar F, Fadıloğlu Ç. Hemşirelikte inovatif alan: evde bakımda telehemşirelik ve telesağlık. Acıbadem Üniversitesi Sağlık Bilimleri Dergisi 2015;6(4):191-5

7. Burdea GC, Jain A, Rabin B, Pellosie R, Golomb M. Long-term hand telerehabilitation on the PlayStation 3: benefits and challenges. In 2011 Annual International Conference of the IEEE Engineering in Medicine and Biology Society.2011: 1835-1838).

8. Nicola K, Waugh J, Charles E, Russell T. The feasibility and concurrent validity of performing the Movement Assessment Battery for Children-2nd Edition via telerehabilitation technology. Research in Developmental Disabilities. 2018; 77: 4048.

9. Pahwa PK, Singh A, Sharma S, Mani S. Efficacy of tele-rehabilitation for the management of physical impairments of the children with cerebral palsy in the inclusive educational settings: a protocol for a systematic review. Indian Journal of Public Health Research \& Development.2020; 11(2): 328-332.

10. Gagnon M, Collins J, Elfassy C, Merlo GM, Marsh J, Sawatzky B, et al. A telerehabilitation intervention for youths with arthrogryposis multiplex congenita: Protocol for a pilot study. JMIR Research Protocols.2020; 9(6): e18688.

11. Buitrago JA, Bolaños AM, Caicedo Bravo E. A motor learning therapeutic intervention for a child with cerebral palsy through a social assistive robot. Disability and Rehabilitation: Assistive Technology.2020; 15(3): 357-362.

12. Chase HP, Pearson JA, Wightman C, Roberts MD, Oderberg AD, Garg SK. Modem transmission of glucose values reduces the costs and need for clinic visits. DiabetesCare.2003; 26(5):1475-1479.

13. Caprì T, Fabio RA, Iannizzotto G, Nucita A. The TCTRS project: A holisticapproachfortelerehabilitationin RettSyndrome. Electronics.2020; 9(3): 491. 14. Gibbs V, Toth-Cohen S. Family-centered occupational therapy and telerehabilitation for children with autism spectrum disorders. Occupational Therapy in Health Care.2011; 25(4): 298-314.

15. Boccalandro EA, Dallari G, Mannucci PM. Telemedicine and telerehabilitation: current and forth coming applications in haemophilia. Blood Transfusion. 2019;17(5): 385 .

16. Rosly MA, Kamaruzaman D, Zulkornain LH, Shamsuddin S, Zahari NI, Yussof,

H. Zorabots: A suitable robot-mediated telerehabilitation interface. In Journal of Physics: Conference Series.2020;1529 (2): 022047.

17. Steinhart S, Raz-Silbiger S, Beeri M, Gilboa Y. Occupation based telerehabilitation intervention for adolescents with Myelomeningocele: A pilot study. Physical \& Occupational Therapy In Pediatrics. 2020: 1-16.

18. Huber M, Rabin B, Docan C, Burdea G, Nwosu ME, Abdelbaky M, Golomb MR. PlayStation 3-based tele-rehabilitation for children with hemiplegia. In 2008 Virtual Rehabilitation.2008: 105-112.

19. Looman WS, Erickson MM, Garwick AW, Cady RG, Kelly A, Pettey C, Finkelstein SM. Meaningful use of data in care coordination by the advanced practice registered nurse: the TeleFamilies Project. Computers, Informatics, Nursing: CIN.2012; 30(12): 649.
20. Rabatin AE, Lynch ME, Severson MC, Brandenburg JE, Driscoll SW. Pediatric telerehabilitation medicine: Making your virtual visits efficient, effective and fun. Journal of pediatric rehabilitation medicine. 2020:1-16 (Preprint).

21. Özden F, Lembarki Y. The ethical necessities and principles in telerehabilitation. Sağlık Hizmetleri ve Eğitimi Dergisi. 2020;3(2): 35-37.

22. Lawend JA, Mohammed BA, Elwasefy SA. Massage therapy program and telephone follow-up effects on anxiety and satisfaction among bronchial asthma children. International Journal of Nursing Didactics. 2020; 10(7): 1-14.

23. Özdemir EA, Örsal Ö. Türkiye'de hemşirelik tezlerindeki tele-hemşirelik uygulamalarının etkisinin incelenmesi: Sistematik derleme. Turkiye Klinikleri J NursSci. 2020;12(4):607-15.

24. Güven ŞT, Dalgiç Aİ, Duman Ö. Evaluation of the efficiency of the web-based epilepsy education program (WEEP) for youth with epilepsy and parents: A randomized controlled trial. Epilepsy\&Behavior. 2020; 111: 107142. 